\title{
Classification of Nepalese Forests and Their Distribution in Protected Areas
}

\section{Tirtha Bahadur Shrestha, Ph.D.1}

\begin{abstract}
Nepal is nature's paradise. It's a small attractive package of nature embracing the rich biological diversity in the tiniest area. One of the nature's gifts to Nepal is its vegetation. The narrow strip of land harbours over 170 parcels of vegetation. The need of categorisation of Nepal's forest type is thus not only needed to acknowledge the rich diversity but also to make it applicable in scientific studies and researches. This paper endeavours to classify the Nepal's forest according to all the rational parameters yet avoiding the strict compartmentalisation which is near to impossible as in the case of natural and life bearing heritage like forest.
\end{abstract}

Key Words: Forest type, Ecological map, Classification, Protected areas

\section{Introduction}

The Himalaya creates a complex mountain ecosystem to give rise to diverse flora, vegetation and forest types. As such classification of vegetation types or forest types becomes very complex too. Mutually exclusive divisions or strict compartmentalization in terms of forest type coverage is not possible nor is desirable because it does not exist in nature. Difficulties in classification are further aggravated due to human intervention and modification of natural vegetation. Some degree of generalization, therefore, becomes necessary, and general adoption of a classification would become more practical. Classifications are aimed for a certain purpose. For the purpose of understanding biodiversity and preparing ecological map of Nepal an integrated classification and nomenclature is arrived on the basis of physiognomy, structure, flora and bioclimate. It has been adopted by Department of Forest (DoF) and has been published under Tree Improvement and Silviculture Component (TISC) Document Series No. 105, 2002.

\section{Basis of Classification}

For a mountain country like Nepal altitudinal limits are most convenient to define ecological zones or life zones. Geographical locations and habitat types provide easy means to differentiate various types within the same or similar physiognomic-structural type. Floristic dominance often provides lead identification and nomenclature of forest types.

The parameters (Table 1)

\section{- Bioclimatic zonation}

Nepal lies just outside of the tropics in the global climatic zonation. However, bioclimatic tropicality extents into it up to an elevation of 1,000 $\mathrm{m}$ altitude. The Sub-

\footnotetext{
Life Member, Nepal Academy, tirtha@infofamily.com.np
} 
tropical zone (1,000-2,000 m), the Temperate zone (2000-3000 m), the Sub-alpine zone (3,000-4,000 m), the Alpine zone (4000-5000 m) and the Nival zone (5,000 and above) appear juxtaposed along mountain slopes. This allows us to specify alpine pasture, sub-alpine mountain oak, temperate juniper, tropical mixed hardwood and so on in the process of vegetation/forest classification.

\section{- Geographical locations}

Geographical specification allows to readily separate out vegetation types such as trans-Himalayan steppe, temperate mountain oak forest or hill Sal forest. Geographical notion of western or eastern type also helps to differentiate forest types such as east Himalayan and west Himalayan, as well as north facing or south facing forests.

- Physiognomy \& structural parameter

Physiognomy is most apparent and hence convenient to describe and delineate forest types such as closed forest, open or dense forests, one-layered or multilayered forests, open woodland or grassland and so on. Structural features such as evergreen, deciduous, coniferous or broadleaved type contribute to aid visual identification. Such features can readily be used as environmental indicators. Besides, the carbon sink capacity of various forest types may also be assessed on the basis of structure. Deciduous forests for example remain inactive in photo-synthesis during winter. As such global carbon sink drops during October-March when northern hemisphere is largely deciduous, and devoid of broadleaves.

\section{- $\quad$ Floristic parameter}

Floristic classification is efficient but it remains too technical when botanical names are used such as Tsuga forest, Schima-Castanopsis forest etc. Latin names may be replaced by common english names such as Hemlock forest for Tsuga forest or Sal forest for Shorea forest based upon local names. Stainton (1972) used mostly botanical names while Forest Statistics (1973) used common english names. Similarly, the Forestry Sector Master Plan (1989) also used common english names such as Birch forest, Fir forest, Khair-Sisso forest and so on.

Table 1: Parameters for Vegetation/Forest Classification

\begin{tabular}{|ll|l|l|}
\hline Bioclimatic Zone & \multicolumn{1}{c|}{ Geography } & \multicolumn{1}{|c|}{ Physiognomy } & \multicolumn{1}{c|}{$\begin{array}{c}\text { Structure \& Life } \\
\text { Form }\end{array}$} \\
\hline Alpine & Trans-Himalayan & Closed forest & Evergreen \\
Sub-alpine & Mountain & Open forest & Deciduous \\
Temperate & Eastern & Grassl ands & Coniferous \\
Sub-tropical & Western & Shrublands & Hardwood \\
Tropical & & & \\
\hline
\end{tabular}




\begin{tabular}{|c|c|c|c|}
\hline $\begin{array}{c}\text { Floristic } \\
\text { Component }\end{array}$ & Dynamics & Habitat & Management \\
\hline $\begin{array}{l}\text { Pine forest } \\
\text { Sal forest } \\
\text { Larix forest }\end{array}$ & $\begin{array}{l}\text { Climax forest } \\
\text { Secondary forest } \\
\text { Plantation forest }\end{array}$ & $\begin{array}{l}\text { Riverine forest } \\
\text { Mountain Oak } \\
\text { forest }\end{array}$ & $\begin{array}{l}\text { Community forest } \\
\text { Private forest } \\
\text { Religious forest } \\
\text { Protected forest }\end{array}$ \\
\hline
\end{tabular}

\section{Classification of Forests/Vegetation}

The government of Nepal has attempted to provide forest classification for the use of forest officers and managers. Currently the wide spread Community Forest User Groups (CFUGs) are using 8 forest types in their operational Plans (Table 2). The Forestry Sector Master Plan (1989) used just 12 forest types where as the Forest Statistics (1973) was already using 21 types. The Land Resource Mapping Project 1986 (LRMP) used only 7 types to cover whole of Nepal. Stainton's Forest of Nepal (1972) elaborated forest types on the basis of detailed floristic studies. The TISC (2000) came up with 37 vegetation types in order to prepare the ecological map of Nepal (2000). It was based upon the extensive work of Dobremez (1972) and his collaborators. Forest types adopted by various contributions are spelled out in Table 2. Details of the TISC Classification (2000) are to be found in TISC Doc. Series No. 105 published in the aegis of NARMSAP (Natural Resource Management Sector Assistance Programme) supported by DANIDA.

Table 2: Forest Types Correspondences

\begin{tabular}{|c|c|c|c|c|c|}
\hline $\begin{array}{c}\text { FUG Forest } \\
\text { T ypes as of } \\
1999\end{array}$ & $\begin{array}{c}\text { Forestry } \\
\text { Sector Master } \\
\text { Plan } 1989\end{array}$ & $\begin{array}{c}\text { Forest Statistics, } \\
1973\end{array}$ & $\begin{array}{l}\text { LRMP, } \\
1986\end{array}$ & Stainton, 1972 & TISC, 2000 \\
\hline \multirow[t]{2}{*}{ Sal Forest } & Sal Forest & Sal & Sal & $\begin{array}{l}\text { Sal } \\
\text { Hills al }\end{array}$ & $\begin{array}{l}\text { Lower Tropical Sal } \\
\text { And Mixed Hardwood } \\
\text { Forest } \\
\text { Hill Sal }\end{array}$ \\
\hline & Khair-Sisso & Khair-Sisso & $\begin{array}{l}\text { Acacia- } \\
\text { Dalbergia }\end{array}$ & $\begin{array}{l}\text { Dalbergia- } \\
\text { Acacia }\end{array}$ & $\begin{array}{l}\text { Sal Zome Riverine } \\
\text { Habitat } \\
\end{array}$ \\
\hline $\begin{array}{l}\text { Sub-Tropical } \\
\text { Deciduous } \\
\text { Forest }\end{array}$ & $\begin{array}{l}\text { TerailLower } \\
\text { Slope Mixed } \\
\text { Hardwood }\end{array}$ & Terai Hardwood & $\begin{array}{l}\text { Tropical } \\
\text { Mixed } \\
\text { Hartwood }\end{array}$ & $\begin{array}{l}\text { Terrinalia } \\
\text { Tropical } \\
\text { Deciduous } \\
\text { Riverine } \\
\text { Forest }\end{array}$ & $\begin{array}{l}\text { Hills Sal } \\
\text { Upper Tropical } \\
\text { Riverine Forest }\end{array}$ \\
\hline & & & & $\begin{array}{l}\text { Tropical } \\
\text { Evergreen } \\
\text { Forest } \\
\text { Sub-Tropical } \\
\text { Evergreen } \\
\text { Forest } \\
\text { Sub-Tropical } \\
\text { Deciduous Hill } \\
\text { Forest }\end{array}$ & $\begin{array}{l}\text { Hill Sal } \\
\text { EugenizOstodes Forest } \\
\text { Hill Sal }\end{array}$ \\
\hline
\end{tabular}




\begin{tabular}{|c|c|c|c|c|c|}
\hline $\begin{array}{l}\text { Schima- } \\
\text { Castanopsis }\end{array}$ & & $\begin{array}{l}\text { LowerSlope Mixed } \\
\text { Hardwood }\end{array}$ & & $\begin{array}{l}\text { Sclima } \\
\text { Castampsis }\end{array}$ & S chima-Castanopsis \\
\hline \multirow[t]{3}{*}{$\begin{array}{l}\text { Alrous } \\
\text { nepalens is }\end{array}$} & & & & Alrus Forest & S chima-Castanopsis \\
\hline & & & & $\begin{array}{l}\text { Sub-Tropical } \\
\text { Serri- } \\
\text { Evergeen Hill } \\
\text { Forest }\end{array}$ & S chima-Castanopsis \\
\hline & & & & $\begin{array}{l}\text { Castanopsis } \\
\text { trbuloides - } \\
\text { C. hys tix } \\
\text { Forest }\end{array}$ & S chima-Castanopsis \\
\hline $\begin{array}{l}\text { Oak- } \\
\text { Rhododendron }\end{array}$ & Oak Forest & Oak Forest & Quercus sps. & $\begin{array}{l}\text { Q. Incara }-Q \text {. } \\
\text { Larnginosa }\end{array}$ & Lower Temperate $\mathrm{Oak}$ \\
\hline \multirow[t]{4}{*}{$\begin{array}{l}\text { Pirous } \\
\text { roxbughtii }\end{array}$} & Chir Pine & Chir Pine & Chir Pine & $\begin{array}{l}\text { Q. Dilata } \\
\text { Pinus } \\
\text { soxburghii } \\
\text { Forest }\end{array}$ & $\begin{array}{l}\text { Lower Temperate Oak } \\
\text { Chir Fine Fonest }\end{array}$ \\
\hline & & Chir Pine $-O a k$ & & & Chir Pine Broadleaved \\
\hline & Chir Pine-S al & Chir Pine-Sal & & & Chir Pine Broadleaved \\
\hline & & $\begin{array}{l}\text { Chir Pine-Lower } \\
\text { Slopes Mixed } \\
\text { Hardwood }\end{array}$ & & & Chir Pine Broadleaved \\
\hline \multirow[t]{4}{*}{$\begin{array}{l}\text { UpperS bpe } \\
\text { Mixed } \\
\text { Hardwood }\end{array}$} & $\begin{array}{l}\text { UpperSlope } \\
\text { Mixed } \\
\text { Hardwood }\end{array}$ & $\begin{array}{l}\text { Upper Slope Mixed } \\
\text { Hardwood }\end{array}$ & & $\begin{array}{l}\text { Upper } \\
\text { Temperate } \\
\text { Mixed } \\
\text { Broadleaved }\end{array}$ & $\begin{array}{l}\text { Deciduous Maple- } \\
\text { Magnolia-Sobus }\end{array}$ \\
\hline & & & & $\begin{array}{l}\text { Upper } \\
\text { Temperate } \\
\text { Mixed } \\
\text { Broadleaved } \\
\text { Lower } \\
\text { Temperate } \\
\text { Mixed } \\
\text { Broadleaved }\end{array}$ & $\begin{array}{l}\text { Mixed Rhododendron- } \\
\text { Maple } \\
\text { Mixed Oak-Laurel }\end{array}$ \\
\hline & & & & Q. Lamellosa & $\begin{array}{l}\text { East Himalayan Oak } \\
\text { Lausel }\end{array}$ \\
\hline & & & & $\begin{array}{l}\text { Lithocarpus } \\
\text { pachyphylla }\end{array}$ & Lithocarpus Forest \\
\hline \multirow[t]{4}{*}{$\begin{array}{l}\text { UpperS bpe } \\
\text { Comifer }\end{array}$} & Bhe Pine & Bhe Pine & Bhe Pine & Pinus excelsa & $\begin{array}{l}\text { Upper Temperate Blue } \\
\text { Pine } \\
\text { Mixed Bhe Pine }-O a k\end{array}$ \\
\hline & & $\begin{array}{l}\text { Hemlock-Upper } \\
\text { Slopes Mixed } \\
\text { Handwood }\end{array}$ & & Abies pindrow & $\begin{array}{l}\text { West Himalayan Fir- } \\
\text { Hemlock }-O a k \\
\text { Fir-Bhe Pine }\end{array}$ \\
\hline & & & & $\begin{array}{l}\text { Picea } \\
\text { snrithiana }\end{array}$ & S pruce \\
\hline & & & & $\begin{array}{l}\text { Cupressus } \\
\text { Rhododendron } \\
\text { Forest } \\
\text { Cedrus }\end{array}$ & $\begin{array}{l}\text { Cypress } \\
\text { Rhododendron Forest } \\
\text { Cedar }\end{array}$ \\
\hline
\end{tabular}




\begin{tabular}{|c|c|c|c|c|}
\hline & & & & $\begin{array}{l}\text { Terrperate Mourtain } \\
\text { Oak }\end{array}$ \\
\hline $\begin{array}{l}\text { Mixed and } \\
\text { Other Corifer }\end{array}$ & Mixed Conifer-Oak & & $\begin{array}{l}\text { Q. } \\
\text { semecarpifolia }\end{array}$ & Mixed Oak-Lanrel \\
\hline & & & & $\begin{array}{l}\text { Mountain Oak- } \\
\text { Rhododendron }\end{array}$ \\
\hline \multirow[t]{3}{*}{$\begin{array}{l}\text { Mixed } \\
\text { Hardwood } \\
\text { Corifer }\end{array}$} & $\begin{array}{l}\text { Hermlock } \\
\text { Mixed Conifer }\end{array}$ & & Tsuga dumosa & Fir-Hemlock-Oak \\
\hline & & & $\begin{array}{l}\text { Juniper } \\
\text { wallichiana }\end{array}$ & \\
\hline & & & Larix & Larch \\
\hline \multirow{4}{*}{$\begin{array}{l}\text { Birch Forest } \\
\text { Fir Forest }\end{array}$} & Birch Forest & \multirow[t]{4}{*}{ Birch Forest } & \multirow{3}{*}{$\begin{array}{l}\text { Betula utilis } \\
\text { Ábies } \\
\text { spectabilis }\end{array}$} & Birch-Rhododendrom \\
\hline & Fir Forest & & & Fir \\
\hline & $\begin{array}{l}\text { Fir-Upper Slopes } \\
\text { Mixed Handwood }\end{array}$ & & & Fir-Oak-Rhododendron \\
\hline & $\mathrm{Fir}\lrcorner \mathrm{Oak}$ & & $\begin{array}{l}\text { Q. } \\
\text { semecarpifolia }\end{array}$ & $\begin{array}{l}\text { Sub-alpine Mountain } \\
\text { Oak }\end{array}$ \\
\hline \multirow{3}{*}{\multicolumn{2}{|c|}{ Birch-Fir }} & & & \\
\hline & & & & $\begin{array}{l}\text { S teppe Suphorbia- } \\
\text { Artimes ia }\end{array}$ \\
\hline & & & & Olea cus pidata \\
\hline & & & Hippophae & $\begin{array}{l}\text { Trans-Himalayan } \\
\text { S teppe }\end{array}$ \\
\hline & & & Popubus & $\begin{array}{l}\text { Trars-Himalayan } \\
\text { S teppe }\end{array}$ \\
\hline & & & $\begin{array}{l}\text { Moist Alpine } \\
\text { Scnub }\end{array}$ & $\begin{array}{l}\text { Dwarf Rhododendron } \\
\text { S cub }\end{array}$ \\
\hline & & & $\begin{array}{l}\text { Dyy Alpine } \\
\text { Scnib }\end{array}$ & $\begin{array}{l}\text { Juniper Scub (Dry } \\
\text { Alpine Scrub) }\end{array}$ \\
\hline & & & $\begin{array}{l}\text { Aesoubrs- } \\
\text { Juglars-Acer }\end{array}$ & $\begin{array}{l}\text { Oak-Horsechestrout- } \\
\text { Maple }\end{array}$ \\
\hline & & & & Alpine Pasture \\
\hline
\end{tabular}

\section{Potential Vegetation Types of Nepal}

The Potential Vegetation Types of Nepal (2000) appears as the ecological map of Nepal (scale 1:25,000 and 1: 100,000). The map was prepared by Tirtha B. Shrestha, Puspa R. Shakya, Damodar P. Joshi and Govinda Joshi. The project was coordinated by Jens-Peter Barnekow Lillesso (Danish Consultant). Data sources were used from eight ecological maps (scale 1:250,000) published by CNRS-Paris authored by the following scientists during 1970 to 1985.

1. Annapurna - Dhaulagiri by Dobremez \& Jest 1970

2. Kathmandu - Everest by Dobremez 1972

3. Central Terai by Dobremez 1973 
4. Biratnagar - Kanchanjunga by Dobremez \& Shakya 1975

5. Jumla - Saipal by Dobremez \& T.B. Shrestha 1978

6. Api - Dhaulagiri by Dobremez 1984

7. Butwal - Mustang by Dobremez, Battner, Jest, Vigny and Joshi 1984

8. Nepalgunj - Dailekh by Dobremez, Joshi and Shrestha 1985

\section{Forest Types in Protected Areas}

Sixteen protected areas of Nepal encompassing 9 National Parks, 3 Wildlife Reserves, 3 Conservation Areas and one Hunting Reserve contain 30 types of vegetation with permanent snow in the nival zone. Table 3 details out their distribution. Figure 1 illustrates vertical range of each protected area as marked by altitudinal limits.

Fig. 1: Altitudinal Range of Protected Areas of Nepal

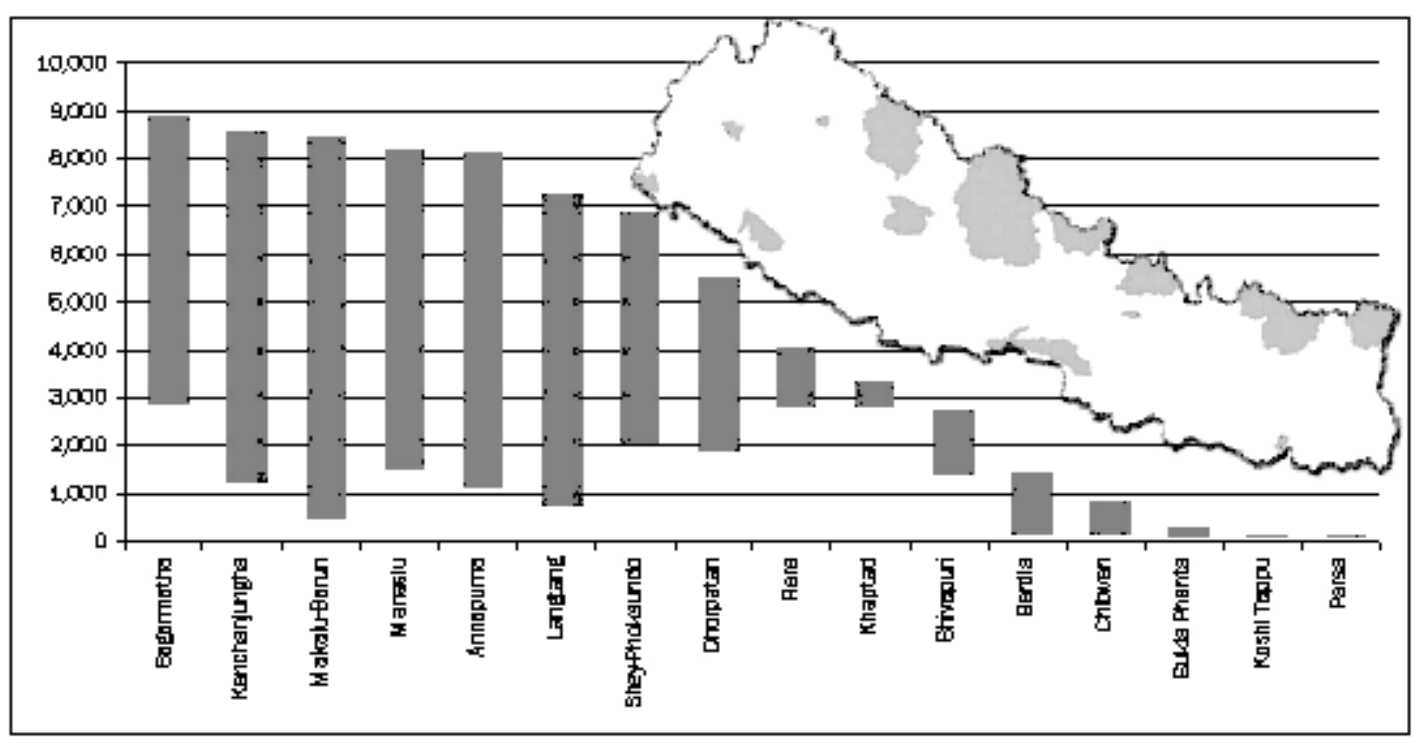

Table 3: Occurrence of Forest Types and Number of Endemic Flowering Plants in Protected Areas

\begin{tabular}{|c|c|c|c|c|c|c|c|c|c|c|c|c|c|c|}
\hline Permanent Snow & $\begin{array}{l}\underbrace{4}_{4} \\
*\end{array}$ & $\begin{array}{l}\text { 舀 } \\
\text { * }\end{array}$ & $\begin{array}{l}\underbrace{4}_{4} \\
* \\
*\end{array}$ & 空 & 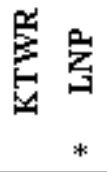 & $\begin{array}{c}\mathcal{4} \\
\text { 鼠 } \\
*\end{array}$ & $\begin{array}{l}\text { 空 } \\
\text { 栬 } \\
\text { * }\end{array}$ & $\mid$\begin{tabular}{l}
\multirow{4}{*}{} \\
$*$ \\
$*$
\end{tabular} & 疍 & 窝 & 芑 & 㝒 & $\begin{array}{c}\text { 完 } \\
*\end{array}$ & 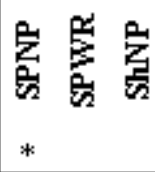 \\
\hline Upper Alpine Meadows & $*$ & $*$ & $*$ & & $*$ & & $*$ & $*$ & & & & & $*$ & $*$ \\
\hline $\begin{array}{l}\text { Trans-Himalayan High Alpine } \\
\text { Vegetation }\end{array}$ & $*$ & $*$ & & & & & & $*$ & & & & & & $*$ \\
\hline $\begin{array}{l}\text { Dry Alpine Scrubs } \\
\text { Moist Alpine Scrubs }\end{array}$ & $*$ & $*$ & $*$ & & $*$ & $*$ & $*$ & $*$ & & & & $*$ & * & $*$ \\
\hline $\begin{array}{l}\text { Trans-Himalayan Upper Caragana } \\
\text { Steppe }\end{array}$ & $*$ & & & & $*$ & & & $*$ & & & & & & $*$ \\
\hline
\end{tabular}




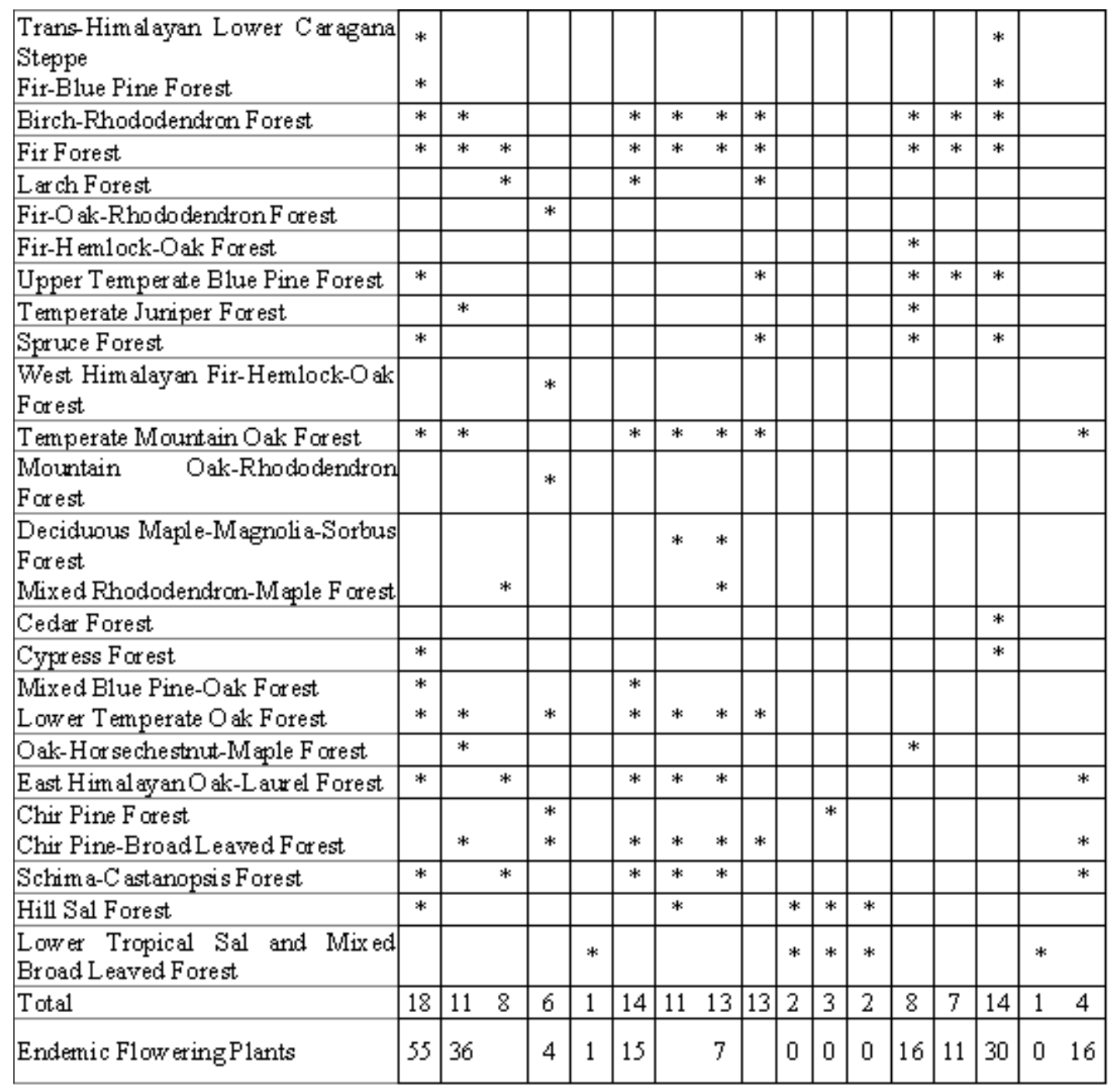

Note: ACA

Annapurna Conservation Area

DHR

Dhorpatan Hunting Reserve

KCA

Kanchanjungha Conservation Area

KNP

Khaptad National Park

KTWR

Koshi Tappur Wildlife Reserve

LNP

Langtang National Park

MBCA

Makalu-Barun Conservation Area (Buffer Zone)

MBNP

Makalu-Barun National Park

MCA

Manaslu Conservation Area

PWR

Parsa Wildlife Reserve 


$\begin{array}{ll}\text { BNP } & \text { Bardia National Park } \\ \text { CNP } & \text { Chitwan National Park } \\ \text { RNP } & \text { Rara National Park } \\ \text { SNP } & \text { Sagarmatha National Park } \\ \text { SPNP } & \text { Shey-Phoksundo National Park } \\ \text { SPWR } & \text { Sukla Phanta Wildlife Reserve } \\ \text { ShNP } & \text { Shivapuri National Park }\end{array}$

\section{Conclusion}

The ecological complexities of Nepal has allowed to identify over 170 parcels of vegetation types in the CNRS vegetation maps (Dobremez et.al 1970 to 1985). They were being reduced to 118 types by the Biodiversity Profiles Project (1995) supported by the GIS unit of ICIMOD. Currently TISC (2000) reduced the 118 types to 36 types excluding the nival zone and the water bodies. The forest type classification through an integration of previous work is expected to be used by forestry practitioners, ecologists and development workers.

\section{Acknowledgements}

This work is the product of a number of scientists of CNRS-Paris and the department of Plant Resources, Govt. of Nepal. I acknowledge all of those who contributed to the vegetation maps produced by CNRS-Paris. I sincerely acknowledge Prof. J.F. Dobremez for his continued support to improve ecological works in Nepal. I duly acknowledge the invaluable support of TISC/NARMSAP staff especially Mr. Prayag Raj Shrestha, Mr. Lokendra Purush Dhakal and Mr. Rabin Shrestha during the entire process of revising and publishing new set of maps and the supporting document "Forest and Vegetation of Nepal". The role of Jens-Peter B. Lilleso, as Project Consultant and Mr. Bo Schultz and Programme Coordinator remained invaluable. Last but not least the support and encouragements of Mr. Chandi P. Shrestha, Secretary, MoFSC and Mr. Dibya D. Bhatta, Director General, DoF are highly acknowledged by the team working for revising the forest and vegetation types of Nepal.

\section{Reference:}

2002, Forest and Vegetation Types of Nepal. TISC Document Series No. 105. Dept of Forest, HMG/NARMSAP, International Year of Mountain Publication, Nepal.

Bhuju, U.R; Shakya, P.R.; Basnet T.B. and Shrestha, S. (2007). Nepal Biodiversity Resource Book - Protected areas, Ramsar Sites and World Heritage Sites. ICIMOD/MOEST, GON/UNEP/Nepalnature.com

BPP (1995a). An Assessment of the Representation of the Terestrial Ecosystems in the Protected Areas System of Nepal. Biodiversity Profiles Project Publication No. 15. Department of National Parks and Wildlife Conservation, Ministry of Forests and Soil Conservation. His Majesty's Government of Nepal, Kathmandu.

Dobremez, J.F. (1976). Le Népal Ecologie et Biogeography, Editions du Centre National de la Recherche Scientifique, Paris, France. 
Dobremez, J.F. (1984). Carte Ecologique du Nepal. Region Dhangarhi-Api 1:250,000. Cahiers Nepalais Documents 10, Centre Nationale de la Recherche Scientifique, Paris, France.

Dobremez, J.F. and Shakya, P.R. (1975). Carte Ecologique du Nepal. IV. Region Biratnagar Kanchenjunga 1:250,000. Doc. Carte Ecol. XVI, pp. 33-48

Dobremez, J.F. and Shrestha, T.B. (1978). Carte Ecologique du Nepal. Region: Jumla-Saipal. Cahiers Nepalais Documents 9, Centre Nationale de la Recherche Scientifique, Paris, France

Dobremez, J.F.; Joshi, D.P.; Shrestha, T.B. and Vigny, F. (1985). Carte Ecologique du Nepal. Region: Nepalganj - Dailekh 1:250,000. Cahiers Nepalais Documents 12, Centre Nationale de la Recherche Scientifique, Paris, France

ICIMOD (1996). GIS Database of Key Indicators of Sustainable Mountain Development in Nepal. Mountain Environment and Natural Resources Information Services (MENRIS), Internal Centre for Integrated Mountain Development.

Kenting Earth Science Limited (1986). Land Resource Mapping Project: Land utilization report appendices two and three. Kenting Earth Science Limited, Canada.

MPFS (1988). Master Plan for Forestry Sector Nepal. Soil Conservation and Watershed Management Plan., HMG Ministry of Forest and Soil Conservation/ADB/FINNIDA, Kathmandu, Nepal.

Shrestha, T.B. (1982). Ecology and Vegetation of North-West Nepal (Karnali Region). Royal Nepal Academy, Kathmandu, Nepal.

Stainton, JDA (1972): Forests of Nepal. John Murray, London.

\section{विजया दशमी तथा शुभ-दिपावली २०६श को उपलक्ष्यमा}

समस्त संरक्षणप्रेमी एवं उपभोक्ता वर्गहरूमा हार्दिक मंगलमय

शुभ-कामना व्यक्त गर्दे वन र वन्यजन्तुको संरक्षणमा

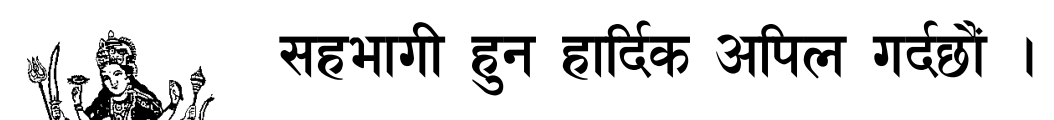

